\title{
EFFICIENT TILE BASED REGION MERGING REMOTE SENSING IMAGE SEGMENTATION
}

\author{
Veena V. Patil ${ }^{1}$, S. G. Shinde ${ }^{2}$ \\ ${ }^{I}$ Department of Electronics and Telecommunication Engineering, TPCT'S College of Engineering, Osmanabad, \\ Maharashtra, India \\ ${ }^{2}$ Professor, Department of Electronics and Telecommunication Engineering, TPCT'S College of Engineering, \\ Osmanabad, Maharashtra, India
}

\begin{abstract}
The remote sensing technologies commonly capture the high resolution images of Earth and transmit them to central process department for further analysis via electromagnetic sensors. Such remote sensing images are stored in digital forms in computers in which further processing is performed for the interpretation purpose. However, processing of high resolution remote sensing large images on computers is challenging due to the large sizes of remote sensing images. For common computers, it is difficult to hold big sized remote sensing datasets for longer time. To solve this problem, segmentation is best solution to save resources for further processing. The remote sensing segmentation method produces the objects of interest from the scene. There is various image segmentation methods reported in recent pasts, but scalability, efficiency, and accuracy is main challenges. In this paper, we proposed tile based image segmentation algorithm. We designed tile-based framework based on region merging algorithm for large images segmentation with identification outcomes. We exploited the stability margin concept for the tile. Additionally, to smooth and fast the process we proposed the robust filtering technique which helps to suppress noise as well as complex data. The filtering approach achieves the improved segmentation results within less or similar processing time. The simulation results shows that proposed method produces more robust results as compared to existing tile based and without tile based methods.
\end{abstract}

Keywords: - Filtering, Image processing, Image Segmentation, Region-Merging, Tilting, Scalability

\section{INTRODUCTION}

The science of remote sensing has arisen as one of the most interesting across the last three decades. The remote sensing is the science of creating regarding objects from measurement; create at distance, without coming into physical contact with the objects under research. Also, remote sensing is states as the sensing of the earth's surface by developing utilize of the properties of electromagnetic wave emitted, emulated or refracted by the sensed objects, for the main intention for increasing the natural resources management, water and land utilize and the preservation of the environment [1]. Earth perception from the space through different remote detecting instruments has given vantage methods for checking land surface flow, characteristic assets administration, and the general condition of the earth itself. Withthe increasing concern for the earth's habitability itself due to the increasing anthropogenic interference with the natural systems, the emphasis on the study of earth system processes has also assumed greater importance [2] [3]. Deriving information about geophysical parameters from the satellite observed reflected or emitted radiation field with appropriate modeling and algorithm development has indeed been a matter of great scientific importance. Advances in optics, devices, signal processing and materials have enabled a quantum jump in imaging and non-imaging sensor systems, providing information about the earth system, hitherto considered not possible from satellites [4].On the other side, the field of image processing is one which has stimulated lots of innovative researches. Image processing is a category of the signal processing is the input of image and the output can either be an image or assets of attributes or parameter associated to the image. The preparing of scene information for self-governing machine recognition is one of the application regions of picture handling [5]. Here, the intrigue concentrated around methodology for separating data from picture in a shape appropriate for computer processing. To accomplish this task, an image needs to be subjected to many image processing steps. Image segmentation is one such task which subdivides an image into its component area or objects. Image segmentation is currently mostly utilized in remote sensing is an efficient order due to possibility of very large resolution visual image. Generally, remote sensing is introduced to the integration and analysis of data about the earth using electromagnetic sensors, which are working from the space borne platform [6] [7].

Remote sensing images are recorded in advanced structures and after that handled by the PCs to deliver pictures for understanding purposes. Pictures are accessible in two structures - photographic film shape and advanced frame. Varieties in the scene qualities are spoken to as varieties in shine on photographic movies [8]. A specific piece of scene reflecting more vitality will seem splendid while an alternate 
piece of a similar scene that reflecting less vitality will seem dark. Advanced picture comprises of discrete picture components called pixels. Related with every pixel is a number denoted to as Digital Number (DN) that delineates the normal brilliance of generally little region inside a scene. The span of this zone impacts the multiplication of subtle elements inside the scene. As the pixel estimate is decreased more scene detail is safeguarded in computerized portrayal [9].

However, the Earth analysis satellites, thus as GeoEye, QuickBird, Pléiades and WorldView supplies VHR (very high resolution) images, which are helpful in applications thus as environmental auditing or natural sources administration. The Pléiades satellites supplies pictures with a ground inspecting separation of $0.5 \mathrm{~m}$ and a spatial scope of $400 \mathrm{~km} 2(40000 \times 40000$ pixels $)$ taking into consideration point by point perception of the Earth surface. Thus, a scene contains billions of pixels, which speaks to a lot of information to process. Managing such amount of information has turned into a testing issue for the remote detecting group due to the constraint of memory accessible on PCs. The established method to take care of this issue is to isolate these vast pictures into littler tiles (rectangular picture subsets of the picture) and process every single one of these tiles autonomously. This function is known as image tiling. There are number methods proposed for remote sensing images segmentation such as object based, texture based, region-merging based, tile based etc. From the literature study, the image tiling helps to process the segmentation faster for high resolution large sizes images, however they failed to cope up well with existing segmentation methods. In this, research paper we investigate the decent tile-based approach depends on the region merging algorithm for huge image segmentation with similar outcomes. The stability margin concept used for the tile based segmentation. Further we proposed the robust filtering technique which helps to suppress noise as well as complex data and hence to improve the segmentation quality and processing speed. In section II, related works discussed. In section III, proposed framework and algorithms presented. In section IV, experimental results and comparative study presented. Finally, the conclusion and future work reported in section $\mathrm{V}$.

\section{RELATED WORKS}

Number of image segmentation methods presented in recent past. In this section, we will take review of some of them in brief. In [1] proposed an unsupervised multi-band technique for scale parameter choice in the multi-scale picture division process, which utilizes phantom point to quantify the ghostly homogeneity of sections. With the expanding scale parameter, unearthly homogeneity of portions diminishes until the point when they coordinate the items in reality. The file of otherworldly homogeneity has been utilized to decide numerous proper scale parameters. The proposed multi-band based division scale parameter determination technique outflanks the single-band based strategy with the better acknowledgment for various land cover protests in various urban scenes.

In [2] introduced a decent image segmentation algorithm for VHR multispectral images using integrated morphological and spectral data. The strategy can be abridged as takes after. Initial, a morphological subsidiary profile has been ascertained from a unique multispectral picture and joined with the unearthly groups to evaluate ghastly morphological qualities of a pixel, which are considered as a paradigm of homogeneity of neighbouring pixels. Picture division was led utilizing a seeded district developing strategy, which has been founded on the seed focuses consequently produced from the angle picture and progressively included and the closeness between a seed pixel and its neighbouring pixels as far as otherworldly morphological qualities. The acquired division result was additionally refined by a locale blending method to produce a last segmentation outcome.

Further the author Zhijian Huang and et al. [3] introduced the decent feature for remote sensing image analysis, is known as MsRS (multi-scale relative salience) feature. It was established by design is procedure of feature value modify with scales. Firstly, the multi-scale perception esteems at each site are acquired by convolved with recursive Gaussian channels for productivity. Also, the multi-scale perceptions esteems are contrasted with the underlying incentive with create the relative striking nature. Finally, the relative remarkable quality between multi-scales is inserting into a solitary element called the MsRS. The scale in MsRS has express spatial significance which was advantageous to pick suitable scale for determined question. In the MsRS delineate, inward of each question turn out to be more predictable, while the difference amongst protest and foundation has been extended. The MsRS can be utilized as preprocessing venture of numerous applications, for example, division. Two condition of-workmanship divisions (the taken into tests and the outcomes demonstrated that it brings change clearly.

In [4] author introduced a recent Region-based Image Segmentation Algorithm, these algorithm based on k-means clustering (RISA), particularly, scheme for remote sensing applications. The algorithm consists of five steps: segment initialization, region growing, k-means clustering, seed production and region integrated. RISA was assessing using a case study concentrating on land-cover classification for two sites: an agricultural region in the RSA and a residential region in Fresno, CA. High spatial determination SPOT 5 and Fast Fledgling satellite symbolism were utilized as a part of the contextual analysis. RISA produced exceedingly homogeneous locales in light of visual assessment. The land-cover arrangement utilizing the RISA-determined picture portions brought about higher exactness than the characterizations utilizing the picture fragments got from the Definiens programming (eCognition) and unique picture pixels in mix with a base separation classifier. 
The Boundary-Constrained Multi-Scale Segmentation (BCMS) techniques innovated in [5]. Initially, adjacent pixels are integrated to produce initial segmentation corresponding to the local area developing scheme. Then, the RAG (Region Adjacency Graph) was constructing based on starting segmentation. Lastly, the neighbourhood common best area consolidating procedure has been connected on Cloth to deliver multi-scale division comes about. Amid the locale consolidating process, a Step-Wise Scale Parameter (SWSP) technique has been proposed to create limit compelled multi-scale division comes about. In addition, with a specific end goal to enhance the precision of protest limits, the property of edge quality was presented as a blending basis. An arrangement of high spatial determination remote detecting pictures was utilized as a part of the investigation, e.g., Brisk Flying creature, Perspective, and airborne picture, to assess the viability of the proposed strategy. The division consequences of BCMS were contrasted and those of the business picture examination programming eCognition.

The novel region merging based segmentation method proposed in [6] called hybrid region merging (HRM). HRM combination the benefits of local-oriented and globaloriented area combined scheme into a merge approach. The comprehensively most-comparative match of areas was utilized to decide the beginning stage of a developing district, which gives an exquisite method to maintain a strategic distance from the issue of beginning stage task and to improve the enhancement capacity for neighbourhood situated locale blending. Amid the area developing system, the combining cycles are compelled inside the nearby region, so the division was quickened and can mirror the neighbourhood setting, as contrasted and the worldwide arranged technique. An arrangement of high-determination remote detecting pictures has been utilized to test the viability of the HRM technique, and three district based remote detecting picture division strategies were received for correlation, including the hierarchical stepwise optimization (HSWO) strategy, the local-mutual best region merging (LMM) strategy, and the multi resolution segmentation (MRS) technique installed in Cognition Developer software.

In [7], researcher a recent approach to multi-scale segmentation of satellite multispectral imagery using edge data. The Canny edge detector was provides to working multispectral edge reorganization. The recognized edge features were then used in multi scale segmentation loop, and the merge methodology for adjoining picture objects has been controlled by a reparability foundation that consolidates edge data with division scale. The centrality of the edge was estimated by contiguous apportioned locales to perform edge appraisal. The present technique has in view of a half-parcel structure, which was made out of three stages: single edge location, isolated pixel gathering, and noteworthy element estimation. The unearthly separation of the half-allotments isolated by the edge was figured, thought about, and coordinated into the edge data.
In [8], the standard is based on the distance spectral variance vector. The region-based segmentation include of a multiple-pass where adjacent segment with a distance lower than a user-stated global threshold are integrated. There is a lower segment size, which impose the creation of segment in region of the higher local variance in the image.

In [9], the researcher investigated an unsupervised regionbased segmentation technique for hyper-spectral information. The initial preprocessing step includes of decreasing the dimension of the hyper-spectral evaluation by working a basic element evaluation. Next, the mean-shift filtering is working to established starting in the image. Lastly, a region-merging procedure using the Bhattacharyya distance.

Currently, the rising of the spatial resolution has made compelling the integration of shape information and spectral data to join in the segments. In [10], the researcher innovated a region-merging algorithm based on spatial and spectral data.

In [11], the researcher innovated a multi-stage segmentation procedure by initially choose the seeds using the gradient of the image. Next, a region-growing model based on spectral and morphological data is supplies using these seeds. Lastly, a region-merging process based on spectra and morphological data is supplies to improve the segment acquire from the region-growing process.

In [12], the researcher innovated a segmentation algorithm integrated spectral and morphological elements to form a ranking of segment for the band of the image. A generic algorithm is then utilized to choose the optimal segments, which according to real object in the scene. The hierarchical segmentation of the image obtained by using a statistical clustering algorithm depends on the Kullback-Leibler divergence.

The region-merging method shows the efficient results for remote sensing image segmentation as most of existing methods takes whole image as input. However, processing large images for image segmentation is time consumption task. Therefore, without loss of generality, another tilebased region-merging segmentation method reported in [13] recently. In [13], tile based region-merging solution to ensure identical outcomes for the segmentation of satellite images of arbitrary size. This method proposed to overcome the previous methods problem where the tiling based approach failed to cope up with region growing segmentation algorithms. However, in this method the possibility of noise suppression and complex data removal is not consider, therefore in this paper we further extend the this method by designing the novel filtering approach to suppress and smooth the color remote sensing image. 


\section{METHODOLOGY}

In this section we present the proposed tile-based region merging algorithm for large scale remote sensing images. In general, split-and-merge algorithm is consists of two steps. First, the technique subdivides the all image into lower regions following a heterogeneity standard. To partition the image, various scheme can be accepted thus as a quad tree partition and a BSP (binary space partition). These two techniques are utilized for partition of image. Another steps, the neighbor area acquire form distributed phase are combined if they are check a similarity standard. These two standard can be depends on the gradient contrast, region statistics, intensity range, texture. The integration of splitting and combined steps enable for the segmentation of arbitrary shapes, which are not strained to vertical or horizontal lines, as occurs if only the distributed step is analysis. Region splitting and merging subdivide an image established into set of arbitrary, disjoint area and then combined or distributed the area in an attempt to fulfil the required condition.

In proposed tile based region-merge approach, stability margin concept has been defined and expressed quantitatively as a function of the number of iterations of the region-merging procedure. The use of stability margin concept in proposed method ensures the segmentation results between with and without tiling. Additionally, we design the dynamic Gaussian filtering to blur the images before segmentation in order to prevent the loss of data and increase the speed of segmentation. As showing in algorithm, the RGB image is first processed using dynamic Gaussian filtering in which the kernel size is selected based on context of input image.

Algorithm: Tile-based Region-Merging Segmentation Inputs

Im: RGB image

$\sigma=0.6$

$\alpha=4$

$\delta=125:$ tile dimension (size of tile)

Output:

Segmented Image

1. Select RGB image

2. double (I)

3. $\mathrm{Ksize}=(\alpha * \sigma)+1$

4. $\operatorname{Im}=$ filter ('Gaussian', Ksize, $\sigma$ )

5. Perform tiling on input image Im

6. $\quad$ Tiles $=$ divide $(\operatorname{Im}, \delta)$

7. Partial Segmentation on Tiles

8. Removal of unstable segments from the graph of tiles
9. Graphs storage

10. Start merging process

11. IF (segments to merge)

12. Add stability margin

13. Partial segmentation

14. Removal of unstable segments

15. Storage

16. ELSE

17. Merge All Graphs

18. END IF

19. IF (all segments merge)

20. Generate final segmentation result

21. ELSE

22. Storage of final graph

23. END IF

\section{PERFORMANCE EVALUATION}

The performance evaluation of proposed method is performed using the MATLAB tool on windows $10 \mathrm{OS}$ with 4 GB RAM and I5 processor. We collected the large size high dimensional real time remote sensing images from the QuickBird scene. Figure 1 showing the original remote sensing image. Figure 2 presents the results of three methods evaluated in this paper such as region-merge without tiling, region-merge with tiling and proposed (filtering based region-merge with tiling) approach.

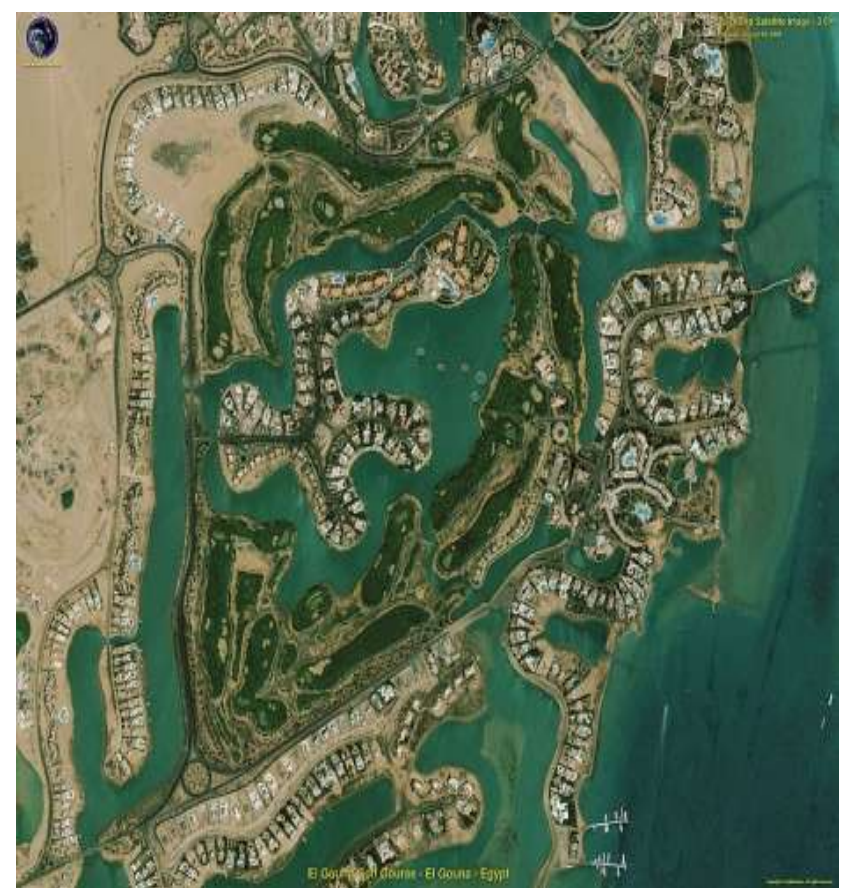

Fig 1: Input test image 1 

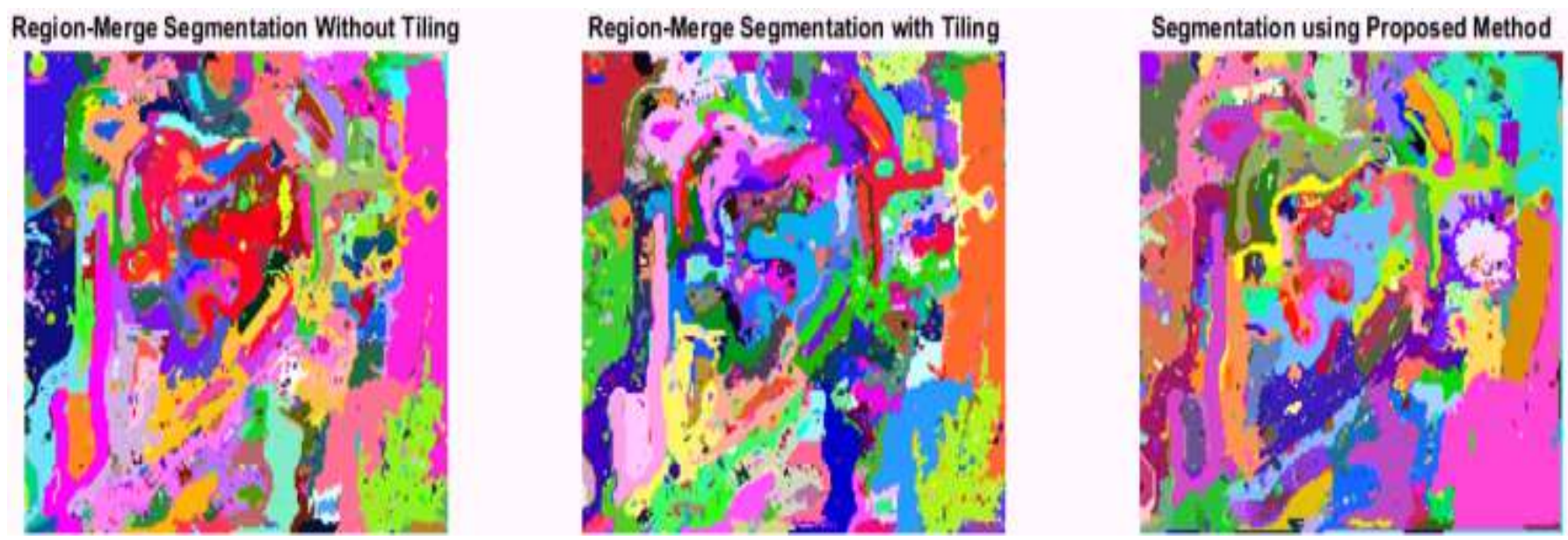

Fig 2: Segmentation Results of test image 1

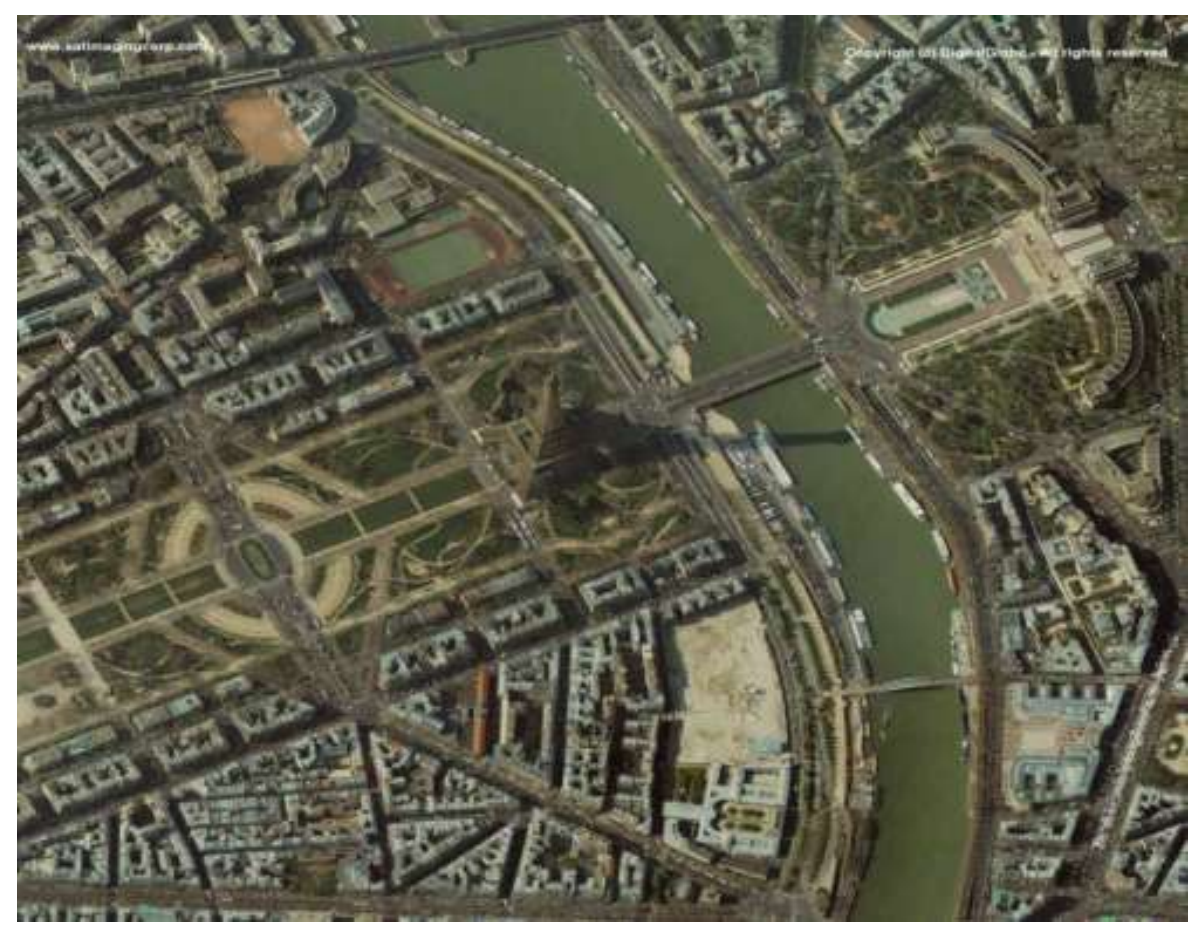

Fig 3: Input test image 2
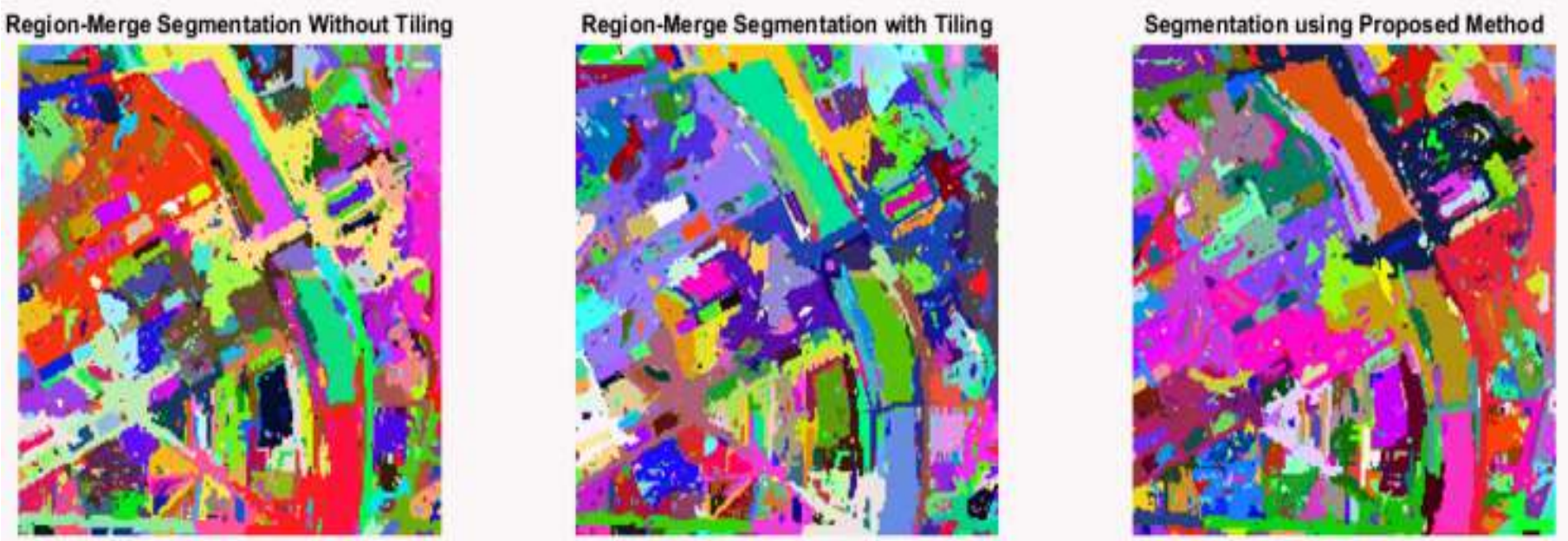

Fig 4: Segmentation Results of test image 2 
Above results showing the proposed method produces the more accurate segmentation as compared to both existing methods. The comparative study among three methods is performed using total computation time required. Figure 5 shows the comparative analysis processing time required for each method for each image. We have evaluated for 3 test images.

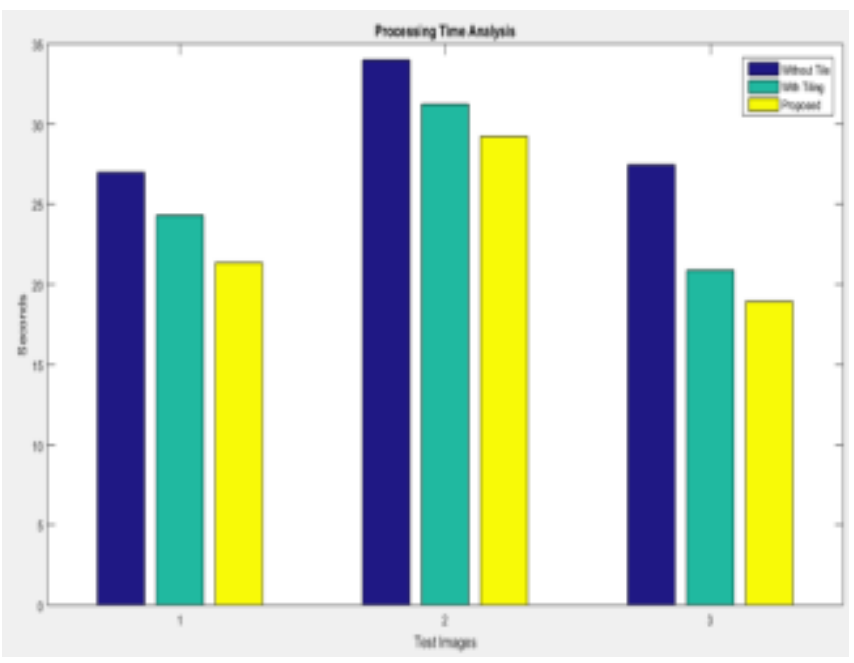

Fig 5: Processing Time comparative study

From the figure 5, it is observed that proposed method not only improves the quality of segmentation but also reduces the required processing time.

\section{CONCLUSION AND FUTURE WORK}

This paper proposed the novel approach for remote sensing images segmentation based on conventional region-merging technique. To improve the scalability and efficiency we modified the region-merging method with tile-based approach and adaptive Gaussian filtering technique. The experimental result shows that proposed method improves the segmentation efficiency as well as minimizes the processing time for large size images for segmentation process. For future work, we like to investigate the use of parallel computing framework further to reduce the computational time.

\section{ACKNOWLEDGMENT}

I would like to express my deep gratitude to my research, Prof. S. G. Shinde as a research guide, for his patient guidance and enthusiastic encouragement of this research work. I would also like to thank the technicians of the laboratory of the E\&TC department for their help in offering me the resources in running the program.

\section{REFERENCES}

[1] Jian Yang, Peijun Li, Yuhong He, "A multi-band approach to unsupervised scale parameter selection for multi-scale image segmentation", Elsevier August 2014.
[2] Jing Liu, Peijun Li , Xue Wang, "A new segmentation method for very high resolution imagery using spectral and morphological information”, Elsevier March 2015.

[3] Zhijian Huang, Jinfang Zhang, Fanjiang Xu, "A novel multi-scale relative salience feature for remote sensing image analysis", Elsevier January 2014.

[4] Zhongwu Wang, John R. Jensen, Jungho Im, “An automatic region-based image segmentation algorithm for remote sensing applications", Elsevier October 2010.

[5] Xueliang Zhang, Pengfeng Xiao, Xiaoqun Song, Jiangfeng She, "Boundary-constrained multi-scale segmentation method for remote sensing images", Elsevier April 2013

[6] Xueliang Zhang, Pengfeng Xiao and et al., "Hybrid region merging method for segmentation of highresolution remote sensing images", 2014.

[7] Jianyu Chen, Delu Pan, Qiankun Zhu and et al., "Edge-Guided Multi scale Segmentation of Satellite Multispectral Imagery", IEEE 2012.

[8] C. Woodcock and V. Harward, "Nested-hierarchical scene models and image segmentation," Int. J. Remote Sens., vol. 13, no. 16, pp. 3167-3187, Nov. 1992.

[9] S. Lee and C. Lee, "Unsupervised segmentation for hyperspectral images using mean shift segmentation," in Proc. SPIE Opt. Eng. Appl., 2010, Art. ID. 781011.

[10] M. Baatz and A. Schäpe, "Multiresolution segmentation: An optimization approach for high quality multi-scale image segmentation," in Angewandte Geographische Informationsverarbeitung XII. Heidelberg, Germany: Wichmann, 2000, pp. 12-23.

[11] K. Segl, S. Roessner, U. Heiden, and H. Kaufmann, "Fusion of spectral and shape features for identification of urban surface cover types using reflective and thermal hyperspectral data," ISPRS J. Photogramm. Remote Sens., vol. 58, no. 1/2, pp. 99112, Jun. 2003.

[12] H. G. Akcay and S. Aksoy, "Automatic detection of geospatial objects using multiple hierarchical segmentations," IEEE Trans. Geosci. Remote Sens., vol. 46, no. 7, pp. 2097-2111, Jul. 2008.

[13] Pierre Lassalle, Jordi Inglada, Julien Michel, Member, IEEE, Manuel Grizonnet, “A Scalable TileBased Framework for Region-Merging Segmentation", IEEE TRANSACTIONS ON GEOSCIENCE AND REMOTE SENSING, VOL. 53, NO. 10, OCTOBER 2015 